\title{
ChemComm
}

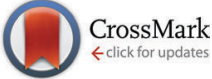

Cite this: Chem. Commun., 2016, 52,5463

Received 23rd January 2016, Accepted 11th March 2016

DOI: $10.1039 / c 6 c c 00679 e$

www.rsc.org/chemcomm

\section{Immobilization of two organometallic complexes into a single cage to construct protein-based microcompartments $\dagger$}

\author{
Basudev Maity, Kazuki Fukumori, Satoshi Abe and Takafumi Ueno*
}

\begin{abstract}
Natural protein-based microcompartments containing multiple enzymes promote cascade reactions within cells. We use the apoferritin protein cage to mimic such biocompartments by immobilizing two organometallic Ir and Pd complexes into the single protein cage. Precise locations of the metals and their accumulation mechanism were studied by X-ray crystallography.
\end{abstract}

Compartmentalization is a common strategy used by living organisms for controlling reaction environments within the cell such as the reactions of metabolic pathways. ${ }^{1}$ The carboxysome is a unique example of a protein-based microcompartment that encapsulates diverse enzymes into a single cage to promote $\mathrm{CO}_{2}$ fixation. ${ }^{2,3}$ Mimicking such compartment systems as new biomimetic materials is important for understanding complex cellular process as well as catalytic applications. In efforts to develop such compartment systems, foreign proteins are incorporated into the protein cage. ${ }^{4-6}$ In particular, Douglas and coworkers have reported an approach where multiple enzymes are packed into a single protein cage of virus-like particles (VLP). There remains the problem that encapsulated enzymes in the cages have lower activity than in free solution as a result of reduced stability, variable structure and other factors. ${ }^{6-8}$

In one of the simpler ways to address this issue, such systems are mimicked by employing artificial metalloenzyme technology. ${ }^{9-12}$ Protein scaffolds provide useful reaction space when occupied by non-natural metal cofactors or metal ions and can promote various catalytic reactions. ${ }^{13-21}$ Recently, artificial metalloenzymes have been used to avoid mutual deactivation of catalysts when combined with biocatalysts for cascade reactions. ${ }^{22-24}$ Ward and coworkers have developed synthetic cascades by combining artificial transfer hydrogenase (ATHase) with oxidase enzymes. ${ }^{22}$

Protein cages that include metal nanoparticles are available for designing cascade reactions in combinations with native

Department of Biomolecular Engineering, Graduate School of Bioscience and Biotechnology, Tokyo Institute of Technology, B-55, 4259 Nagatsuta-cho, Midori-ku, Yokohama 226-8501, Japan. E-mail: tueno@bio.titech.ac.jp

$\dagger$ Electronic supplementary information (ESI) available: Experimental procedures, X-ray crystallographic data. See DOI: 10.1039/c6cc00679e enzymes or metal complexes in buffer solutions. ${ }^{23,24}$ Although such combinations of biocatalysts and artificial metalloenzymes promote cascade reactions, it remains challenging to isolate each catalytic reaction within a single cage in a manner similar to reactions occurring in natural protein-based microcompartments. In this work, our objective was to construct a single protein cage containing multiple metal complex catalyst species to promote tandem reactions. We selected ferritin, a natural iron storage protein, because this self-assembled 24-mer protein provides a spherical cavity with an internal diameter of $8 \mathrm{~nm}$ that is stable over a wide $\mathrm{pH}$ range $(2-11)$ and at temperatures up to $80{ }^{\circ} \mathrm{C} .{ }^{25}$ Individual incorporation of various metal complexes into the apo-ferritin cage have been investigated in terms of the metal accumulation process, catalysis and drug delivery. ${ }^{18,26-33} \mathrm{We}$ are now interested in whether two different organometallic complexes can be immobilized simultaneously on the interior surface of a single apo-ferritin cage to develop an artificial protein compartment (Fig. 1). Here we describe the preparation of apo-ferritin containing two different IrCp* and $\operatorname{Pd}($ allyl) organometallic moieties and structural characterization of the resulting composite by X-ray crystallography. Catalytic reactions such as Suzuki-Miyaura coupling and hydrogenation reaction by the single protein cage containing dual organometallics are also described.

Immobilization of two different metal complexes within the single protein cage was performed by introducing the $\left[\operatorname{IrCp}^{*} \mathrm{Cl}_{2}\right]_{2}$ complex into the recombinant L-chain apo-ferritin from horse liver (apo-rHLFr) followed by introduction of the $[\mathrm{Pd}(\text { allyl }) \mathrm{Cl}]_{2}$ complex. The IrCp* immobilization reaction was carried out by stirring apo-rHLFr $(10 \mu \mathrm{M}$ in $50 \mathrm{mM}$ Tris-HCl (pH 8.0), $0.15 \mathrm{M} \mathrm{NaCl}$ ) with 85 equiv. of $\left[\operatorname{IrCp}^{*} \mathrm{Cl}_{2}\right]_{2}$ for $1 \mathrm{~h}$ at $50{ }^{\circ} \mathrm{C}$. After reaction, the mixture was dialyzed against $0.15 \mathrm{M} \mathrm{NaCl}$ to remove unbound $\mathrm{IrCp}^{*}$ moieties and finally the ferritin protein cage containing IrCp* complexes (IrCp*-apo-rHLFr) was purified by gel filtration (Superdex G200). The number of IrCp* moieties accumulated in IrCp* apo-rHLFr was estimated using inductively coupled plasma-mass spectrometry (ICP-MS) and the bicinchoninic acid (BCA) assay. These analyses suggest that a total of $37 \pm 4$ Ir atoms 
(a)

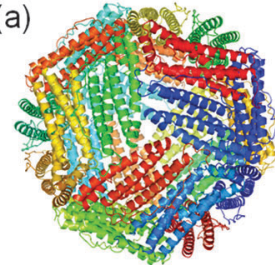

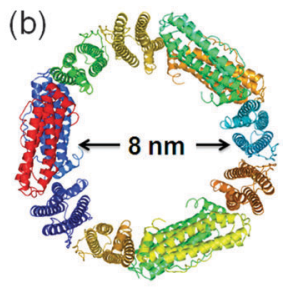

(c)

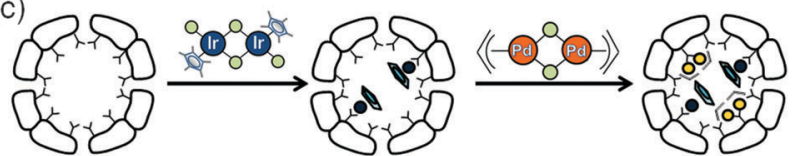

Fig. 1 (a) Schematic representation of entire apo-ferritin (PDB: 1DAT) structure. (b) Cross section of inner cavity. (c) Reaction scheme for immobilization of $\left[\mathrm{IrCp}{ }^{*} \mathrm{Cl}_{2}\right]_{2}$ and $[\mathrm{Pd}(a \mid l y l) C l]_{2}$ complexes into single protein cage.

are accumulated per apo-rHLFr cage. To determine the precise locations of the IrCp* moieties in the apo-rHLFr cage, we crystallized the IrCp*apo-rHLFr species using the hanging drop vapor diffusion method as reported previously. ${ }^{18,26}$ The crystal structure was refined to $1.56 \AA$ resolution as shown in Fig. 2a. Selected crystallographic data and refinement statistics are listed in Table S1 (ESI $\dagger$ ). The positions of the IrCp* moieties were determined from the anomalous difference Fourier maps (see ESI $\dagger$ ). In the metal accumulation centre, two different IrCp* binding sites are present. A large ellipsoidally formed anomalous density is observed near His49. It is expected that several conformers of $\operatorname{Ir}-\mathrm{N}^{\varepsilon}$ (His49) exist at the binding sites in the 24-mer cage of apo-rHLFr. Three different $\operatorname{IrCp}^{*}$ binding positions (Ir1A, Ir1B and Ir1C) were a)

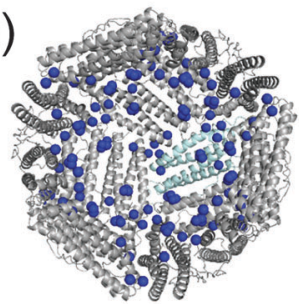

b)

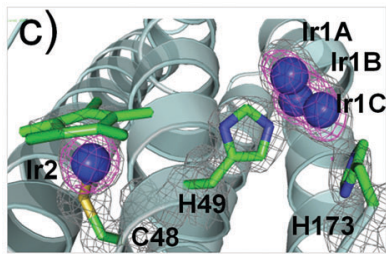

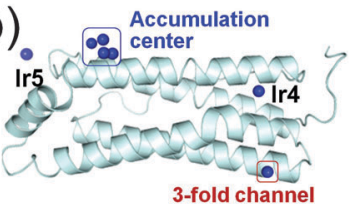

d)

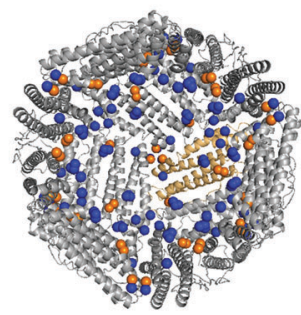

e)
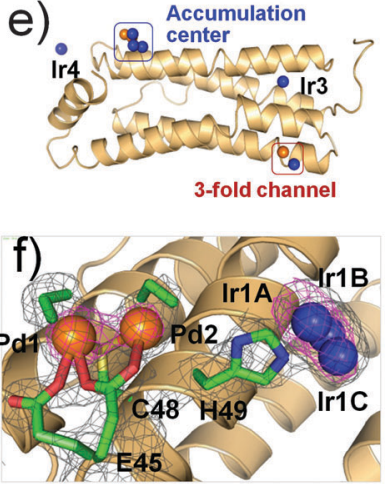

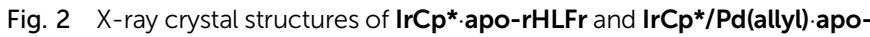
rHLFr showing entire interior structures ( $a$ and $d)$, monomer structures ( $b$ and e) and metal accumulation sites (c and f), respectively. Anomalous difference Fourier maps (pink) at $4.0 \sigma$ indicate the positions of Ir (blue spheres) and $\mathrm{Pd}$ atoms (orange spheres). The $2\left|F_{\mathrm{o}}\right|-\left|F_{c}\right|$ electron density maps at $1.0 \sigma$ are coloured in gray. modelled with three possible orientations of the imidazole moiety with a reasonable Ir-N distance range of 2.08-2.45 (Fig. 2c and Fig. S1, ESI $\dagger$ ). The adjacent His173 was found to coordinate Ir1C with an Ir1C- $\mathrm{N}^{\varepsilon}$ (His173) distance of $2.40 \AA$. The total combined occupancy of Ir1A, Ir1B and Ir1C is 0.8 , although the electron density corresponding to the $\mathrm{Cp}^{*}$ ligand was not observed in the vicinity of the Ir ions due to the low occupancy of each Ir metal. The other IrCp* moiety (Ir2) was observed as bound to $\mathrm{S}^{\gamma}$ (Cys48) with a bond distance of $2.35 \AA$ and an occupancy of 0.4 (Fig. 2c). At the 3-fold channel, there are three IrCp* binding sites from 3 different monomers (Fig. S2, ESI $\dagger$ ). Ir3 is ligated by His114 with a bond distance of $2.13 \AA$ and occupancy of 0.3 . Phe128 coordinates to Ir4 (occupancy 0.2 ) with an Ir4-C ${ }^{\varepsilon}$ (Phe128) distance of $2.12 \AA$, which is comparable to the literature (Fig. S3, ESI $\dagger$ ). ${ }^{34}$ The other binding site for Ir5 was observed at the 4-fold axis channel with occupancy of 0.2 (Fig. S3, ESI†). Although the total number of Ir binding sites observed in the crystal structure is 120 , the sum of Ir atoms estimated from all of the occupancies is 45 in a single cage of apo-rHLFr. This value is consistent with that obtained from quantitative analyses (ICP/BCA) $(37 \pm 4)$. Thus, IrCp* moieties are expected to tend to coordinate His residues at the accumulation center and the 3-fold channel.

After immobilization of IrCp* into the apo-rHLFr cage and structural characterization, we focused on introducing organometallic $[\mathrm{Pd}(\mathrm{allyl}) \mathrm{Cl}]_{2}$ complexes into the $\mathbf{I r C p}{ }^{*}$.apo-rHLFr structure to accumulate different metal complexes inside the single protein cage. The deposition of $\mathrm{Pd}(\mathrm{allyl})$ was carried out by stirring 100 equiv. of $[\operatorname{Pd}(\text { allyl }) \mathrm{Cl}]_{2}$ with $\mathbf{I r C p}$ p $^{*}$ apo-rHLFr $(5 \mu \mathrm{M})$ in $0.15 \mathrm{M}$ $\mathrm{NaCl}$ at RT for $1 \mathrm{~h}$. The mixture was purified by dialysis in $0.15 \mathrm{M}$ $\mathrm{NaCl}$ followed by gel filtration (Superdex G200) to remove excess unbound Pd(allyl). The ICP-MS/BCA analyses indicate a total of $35 \pm 3$ Ir and $95 \pm 6 \mathrm{Pd}$ atoms present per apo-rHLFr cage. Interestingly, the number of IrCp* moieties remained almost the same as that of IrCp*apo-rHLFr. This indicates that $\operatorname{Pd}($ allyl) binds at different locations than IrCp*. The crystal structure of ferritin cage containing $\operatorname{IrCp}^{*}$ and $\mathrm{Pd}($ allyl) complexes $\left(\mathbf{I r C p} \mathbf{p}^{*} / \mathbf{P d}(\mathbf{a l l y l}) \cdot \mathbf{a p o}-\mathbf{r H L F r}\right)$ was refined to $1.87 \AA$ resolution as shown in Fig. 2d. Selected crystallographic data and refinement statistics are listed in Table S1 (ESI $\dagger$ ). The overall spherical structure of the apo-rHLFr cage is conserved in the presence of both $\mathrm{IrCp}^{*}$ and $\mathrm{Pd}(\mathrm{allyl})$ complexes because of the root mean square deviation (rmsd) of the $\mathrm{C}^{\alpha}$ atoms from aporHLFr (0.23). The positions of Ir are distinguished from those of Pd metals by comparing the anomalous maps (see ESI $\dagger$ ). The Ir binding sites are identical to those of $\operatorname{IrCp}^{*}$-apo-rHLFr with similar occupancy values, except for the absence of IrCp* bound to $\mathrm{S}^{\gamma}$ (Cys48). The Pd(allyl) moieties were identified by comparing our previously reported structures of Pd(allyl).apo-rHLFr. ${ }^{26}$ At the accumulation centre, there is one $\operatorname{IrCp}^{*}$ binding site and two Pd(allyl) binding sites (Fig. 2f). The coordination of IrCp* at His49 remained the same as in IrCp*apo-rHLFr with total occupancy of 1.0. The electron density corresponding to His173, which coordinates to Ir1C in IrCp*.apo-rHLFr, was not determined due to insufficient density (Fig. $2 \mathrm{c}$ and f). The observed Ir- $\mathrm{N}^{\varepsilon}$ (His49) bond distances are 2.07-2.61 $\AA$. The IrCp* binding 
site at Cys48 in IrCp*apo-rHLFr was replaced by two Pd(allyl)

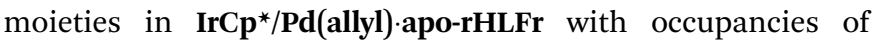
1.0 (Pd1) and $0.7(\mathrm{Pd} 2)$, respectively. This suggests that $\mathrm{Pd}($ allyl) tends to coordinate to Cys. Glu45 showed dual conformations that coordinate to $\mathrm{Pd}($ allyl). The $\mathrm{Pd}($ allyl) moieties maintain a $S^{\gamma}$ (Cys48) bridged dinuclear structure supported by the carboxyl group of one of the adjacent Glu45 conformers (Fig. 2f). The other conformer of Glu45 coordinates to Pd1, which has higher occupancy than Pd2. Both the Pd1 and Pd2 maintain a squareplanar geometry with an allyl ligand, $\mathrm{S}^{\gamma}$ (Cys48) and $\mathrm{O}^{\delta}$ (Glu45). The structure is similar to the reported dinuclear $\mathrm{Pd}($ allyl) complexes. ${ }^{26,35}$ The observed Pd1-Pd2 distance was $2.95 \AA$. The Pd1-S ${ }^{\gamma}(\mathrm{Cys} 48)$ and $\mathrm{Pd} 2-\mathrm{S}^{\gamma}(\mathrm{Cys} 48)$ distances were $2.33 \AA$ and $2.76 \AA$, respectively, whereas the $\mathrm{Pd} 1-\mathrm{O}^{\delta} 1(\mathrm{Glu} 45)$ and Pd2- $\mathrm{O}^{\delta} 2(\mathrm{Glu} 45)$ distances are $2.40 \AA$ and $2.51 \AA$, respectively. The Pd1-O ${ }^{\delta} 1(\mathrm{Glu} 45)$ distance of alternate Glu45 conformer was $2.29 \AA$. The anomalous intensity map at the 3 -fold channel suggests that both Ir 2 and Pd3 are present with occupancies of 0.4 and 0.6, respectively (Fig. S2 and S4, ESI $\dagger$ ). His114 coordinates to Ir2 at an $\operatorname{Ir}-\mathrm{N}^{\varepsilon}($ His114) distance of $2.71 \AA$ A. The short distance between the imidazole (His114) and Pd3 indicates the possibility of double conformations of His114 because both metals have occupancy of $\sim 0.5$ (Fig. S4, ESI $\dagger$ ). The observed distance between the $\operatorname{Pd} 3$ and $\mathrm{N}^{\varepsilon}$ of the alternate conformation of His114 is $2.42 \AA$. The structure indicates that Cys126 coordinates to $\mathrm{Pd} 3$ when it is ligated by the respective His114 conformation (Fig. S4, ESI $\dagger$ ). The observed Pd3-S ${ }^{\gamma}$ (Cys126) and Ir2-S $\mathrm{S}^{\gamma}$ (Cys126) distances are $2.55 \AA$ and $3.19 \AA$, respectively. The Ir binding sites at Phe128 and the 4-fold channel remained the same as in IrCp*apo-rHLFr (Fig. S3, ESI $\dagger$ ). The total number of Ir and Pd binding sites per apo-rHLFr cage observed from the crystal structures is 96 and 72, respectively. The sum of the Ir ions estimated from all occupancies is 40 and this is consistent with the number $(35 \pm 3)$ obtained from the ICP/BCA assay. However, the number of Pd ions $(95 \pm 6)$ determined from the quantitative analysis is higher than the total number of Pd binding sites (72). This suggests that several Pd(allyl) complexes are located at nonspecific binding sites as reported previously. $^{36}$

Interestingly, although we attempted to prepare the heterometallic composite in a reversed procedure with $[\mathrm{Pd}(\text { allyl }) \mathrm{Cl}]_{2}$ incorporated first, followed by $\left[\operatorname{IrCp}^{*} \mathrm{Cl}_{2}\right]_{2}$ and the reaction was unsuccessful because of the precipitation. We suspect that because Pd(allyl) forms a stable dinuclear structure involving coordination to His49 or His114 in Pd(allyl) apo-rHLFr, the $\mathrm{IrCp}^{*}$ deposition in the second step may not be able to displace $\mathrm{Pd}$ and, as a result, random immobilization and precipitation occur. $^{26}$ This observation suggests that the particular reaction sequence is important for immobilization of different metal complexes into a single protein cage. The process of accumulation of $\mathrm{Pd}($ allyl $)$ was understood when 50 equiv. of $[\mathrm{Pd}(\text { allyl }) \mathrm{Cl}]_{2}$ was reacted with IrCp $^{*}$-apo-rHLFr and determined the intermediate structure (IrCp*/I-Pd(allyl)-apo-rHLFr) (Fig. S5, ESI $\dagger$ ). The positions of the $\operatorname{IrCp}^{*}$ and $\operatorname{Pd}($ allyl) in the intermediate structure remained the same as in $\operatorname{IrCp}^{*} / \mathbf{P d}($ allyl).apo-rHLFr except for the coordination structures and occupancies of Pd.
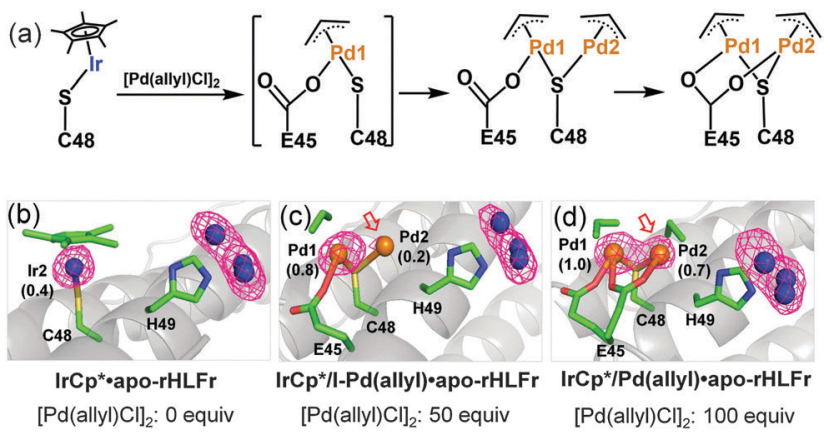

Fig. 3 (a) Possible mechanism of accumulation for Pd(allyl) into IrCp*.aporHLFr. Corresponding crystal structures are shown in (b)-(d). The occupancies of Pd and Ir bounded by Cys48 are shown in parentheses. The anomalous maps at $4 \sigma$ are shown in pink. The arrows show the increment of anomalous density corresponding to Pd(allyl) accumulation.

The occupancies of $\mathrm{Pd} 1$ and $\mathrm{Pd} 2$ are 0.8 and 0.2 , respectively, whereas in $\operatorname{IrCp}^{*} / \mathbf{P d}($ allyl)-apo-rHLFr they are 1.0 and 0.7 , respectively. This suggests that $\operatorname{Pd} 1$ accumulates first and then Pd2, which is confirmed from the comparison of crystal structures (Fig. 3b, c and d). Pd1 is bounded by Cys48 and one carboxylate oxygen atom of Glu45 whereas $\mathrm{Pd} 2$ is bounded by only Cys48 giving long distance of 3.0 A. The results suggest that the Pd(allyl) immobilization at the metal accumulation site occurs in two steps (Fig. 3a). First, the IrCp* bounded by Cys48 is replaced by $\mathrm{Pd} 1$ and stabilized by one of the carboxylate oxygen of Glu45. In the next step, another new Pd(allyl) (Pd2) binds to Cys48 to form a dinuclear structure as reported previously. ${ }^{36}$ The phenomenon was evidenced from the low occupancy of $\operatorname{Pd} 2(0.2)$ compared to Pd1(1.0), which increased to 0.7 when 100 equiv. of Pd(allyl) was used (Fig. 3c and d). During the accumulation process of $\mathrm{Pd} 2$, flexible Glu45 changes its conformation to stabilize the dinuclear Pd structure with two carboxylate oxygen atoms. Similarly, in the 3 -fold channel the occupancy of Pd increases from 0.2 to 0.6 when 100 equivalent of $\mathrm{Pd}($ allyl) was used (Fig. S6, ESI $\dagger$ ). The results suggest that the accumulation of Pd(allyl) occurs stepwise and does not disturb the IrCp* except at Cys48. The use of higher equivalent of Pd(allyl) to saturate the Pd binding sites was avoided due to parallel nonspecific binding.

After immobilization of $\operatorname{IrCp}^{*}$ and $\mathrm{Pd}($ allyl) complexes into a single apo-rHLFr cage, we tested the catalytic activities using 4-iodoacetophenone as a substrate for Suzuki-Miyaura coupling and hydrogenation reactions in aqueous medium at $40{ }^{\circ} \mathrm{C}$. The formation of biphenyl ethanol (yield $\sim 10 \%$ ), which was detected by HPLC, suggests the promotion of catalytic tandem reactions by $\operatorname{IrCp}^{*} / \mathbf{P d}($ allyl).apo-rHLFr (Fig. S7 and S8, ESI $\dagger$ ). The observed enantioselectivity was $\sim 15 \%(S)$. Thus the single protein cage catalyzes both reactions with formation of a mixture of products (Table S3, ESI $\dagger$ ). Size exclusion chromatography and dynamic light scattering of the reaction mixture indicate that the spherical cage structure of ferritin is maintained during the reaction (Fig. S9 and S10, $\mathrm{ESI} \dagger$ ). A similar reaction using the mixture of $\left[\mathrm{IrCp}^{*} \mathrm{Cl}_{2}\right]_{2}$ and $[\mathrm{Pd}(\text { allyl }) \mathrm{Cl}]_{2}$ gives only the coupling product $(\sim 85 \%)$. This indicates that the unique protein environment stabilizes and activates the $\operatorname{IrCp}^{*}$ moiety with histidine 
coordination for catalytic hydrogenation reaction. We also performed the reaction using the mixture of IrCp*apo-rHLFr and Pd(allyl)-apo-rHLFr, which gives similar yield for biphenyl ethanol as for IrCp*/Pd(allyl)-apo-rHLFr. It is expected that the apo-rHLFr cage is not diffusion limiting for small substrates and the intermediate between the cages as observed for VLP containing enzymes. ${ }^{7}$ The results demonstrate the possibility of achieving tandem reactions within a single protein cage by immobilizing different metal catalysts. Optimization of the catalytic reaction to improve activity and selectivity is now underway.

In summary, we have demonstrated immobilization of two different organometallic IrCp* and $\mathrm{Pd}($ allyl) complexes into a single apo-rHLFr cage for promotion of tandem reactions. The $\mathrm{X}$-ray crystal structure analyses suggest that $\mathrm{IrCp}^{*}$ tends to bind to His whereas Pd(allyl) forms a dinuclear structure at Cys48. Both metal complexes are catalytically active within the cage. There is scope for improving catalytic efficiency. Incorporation of multiple complexes within a cage based on principles of coordination chemistry is expected to be a useful strategy for mimicking protein-based microcompartments.

The work is supported by Next-Generation World-Leading Researchers (Grant LR019 to T. U.) for Scientific Research on Innovative Areas (26102513), Ministry of Education, Culture, Sports, Science and Technology, and the Tokuyama Science Foundation, Japan. We thank Mr T. Hikage (Nagoya University, Japan) for X-ray data measurement. Synchrotron radiation experiments were conducted under the approval of 2013A1323, 2013B1262, 2014A1165, 2014A1182 at SPring-8 BL38B1 with the support of members. BM acknowledges Japan Society for the Promotion of Science (JSPS) for research fellowship.

\section{Notes and references}

1 A. H. Chen and P. A. Silver, Trends Cell Biol., 2012, 22, 662-670.

2 T. O. Yeates, C. A. Kerfeld, S. Heinhorst, G. C. Cannon and J. M. Shively, Nat. Rev. Microbiol., 2008, 6, 681-691.

3 S. Tanaka, C. A. Kerfeld, M. R. Sawaya, F. Cai, S. Heinhorst, G. C. Cannon and T. O. Yeates, Science, 2008, 319, 1083-1086.

4 M. Comellas-Aragones, H. Engelkamp, V. I. Claessen, N. A. J. M. Sommerdijk, A. E. Rowan, P. C. M. Christianen, J. C. Maan, B. J. M. Verduin, J. J. L. M. Cornelissen and R. J. M. Nolte, Nat. Nanotechnol., 2007, 2, 635-639.

5 B. Wörsdörfer, K. J. Woycechowsky and D. Hilvert, Science, 2011, 331, 589-592.

6 D. P. Patterson, P. E. Prevelige and T. Douglas, ACS Nano, 2012, 6, 5000-5009.

7 D. P. Patterson, B. Schwarz, R. S. Waters, T. Gedeon and T. Douglas, ACS Chem. Biol., 2014, 9, 359-365.

8 I. J. Minten, V. I. Claessen, K. Blank, A. E. Rowan, R. J. M. Nolte and J. J. L. M. Cornelissen, Chem. Sci., 2011, 2, 358-362.
9 S. Kang and T. Douglas, Science, 2010, 327, 42-43.

10 F. Yu, V. M. Cangelosi, M. L. Zastrow, M. Tegoni, J. S. Plegaria, A. G. Tebo, C. S. Mocny, L. Ruckthong, H. Qayyum and V. L. Pecoraro, Chem. Rev., 2014, 114, 3495-3578.

11 N. J. M. Sanghamitra and T. Ueno, Chem. Commun., 2013, 49, 4114-4126.

12 T. R. Ward, Acc. Chem. Res., 2011, 44, 47-57.

13 T. K. Hyster, L. Knörr, T. R. Ward and T. Rovis, Science, 2012, 338, 500-503.

14 A. Onoda, Y. Kihara, K. Fukumoto, Y. Sano and T. Hayashi, ACS Catal., 2014, 4, 2645-2648.

15 J. Bos, F. Fusetti, A. J. M. Driessen and G. Roelfes, Angew. Chem., Int. Ed., 2012, 51, 7472-7475.

16 M. L. Zastrow, A. F. A. Peacock, J. A. Stuckey and V. L. Pecoraro, Nat. Chem., 2011, 4, 118-123.

17 Y. Lu, N. Yeung, N. Sieracki and N. M. Marshall, Nature, 2009, 460, 855-862.

18 S. Abe, K. Hirata, T. Ueno, K. Morino, N. Shimizu, M. Yamamoto, M. Takata, E. Yashima and Y. Watanabe, J. Am. Chem. Soc., 2009, 131, 6958-6960.

19 N. Yokoi, Y. Miura, C.-Y. Huang, N. Takatani, H. Inaba, T. Koshiyama, S. Kanamaru, F. Arisaka, Y. Watanabe, S. Kitagawa and T. Ueno, Chem. Commun., 2011, 47, 2074-2076.

20 J. M. Chalker, C. S. C. Wood and B. G. Davis, J. Am. Chem. Soc., 2009, 131, 16346-16347.

21 J. M. McFarland and M. B. Francis, J. Am. Chem. Soc., 2005, 127, 13490-13491.

22 V. Köhler, Y. M. Wilson, M. Dürrenberger, D. Ghislieri, E. Churakova, T. Quinto, L. Knörr, D. Häussinger, F. Hollmann, N. J. Turner and T. R. Ward, Nat. Chem., 2013, 5, 93-99.

23 A. Prastaro, P. Ceci, E. Chiancone, A. Boffi, R. Cirilli, M. Colone, G. Fabrizi, A. Stringaro and S. Cacchi, Green Chem., 2009, 11, 1929-1932.

24 Z. Varpness, J. W. Peters, M. Young and T. Douglas, Nano Lett., 2005, 5, 2306-2309.

25 X. Liu and E. C. Theil, Acc. Chem. Res., 2005, 38, 167-175.

26 S. Abe, J. Niemeyer, M. Abe, Y. Takezawa, T. Ueno, T. Hikage, G. Erker and Y. Watanabe, J. Am. Chem. Soc., 2008, 130, 10512-10514.

27 K. Fujita, Y. Tanaka, T. Sho, S. Ozeki, S. Abe, T. Hikage, T. Kuchimaru, S. Kizaka-Kondoh and T. Ueno, J. Am. Chem. Soc., 2014, 136, 16902-16908.

28 K. Fujita, Y. Tanaka, S. Abe and T. Ueno, Angew. Chem., Int. Ed., 2016, 55, 1056-1060.

29 Z. Yang, X. Wang, H. Diao, J. Zhang, H. Li, H. Sun and Z. Guo, Chem. Commun., 2007, 3453-3455.

30 S. Aime, L. Frullano and S. Geninatti Crich, Angew. Chem., Int. Ed., 2002, 41, 1017-1019.

31 B. Maity, K. Fujita and T. Ueno, Curr. Opin. Chem. Biol., 2015, 25, 88-97.

32 C. D. Spicer and B. G. Davis, Chem. Commun., 2011, 47, 1698-1700.

33 T. Granier, G. Comberton, B. Gallois, B. Langlois d'Estaintot, A. Dautant, R. R. Crichton and G. Précigoux, Proteins: Struct., Funct., Bioinf., 1998, 31, 477-485.

34 R. P. Hughes, R. B. Laritchev, A. Williamson, C. D. Incarvito, L. N. Zakharov and A. L. Rheingold, Organometallics, 2003, 22, 2134-2141.

35 R. o. Redón, R. Cramer, S. Bernès, D. Morales and H. Torrens, Polyhedron, 2001, 20, 3119-3125.

36 S. Abe, T. Hikage, Y. Watanabe, S. Kitagawa and T. Ueno, Inorg. Chem., 2010, 49, 6967-6973. 